\title{
Acute Gastric Dilation after Catheter Ablation
}

\author{
Tomoya Sato, Takao Konishi, Rui Kamada and Toshihisa Anzai
}

Key words: gastric dilation, catheter ablation

(Intern Med 59: 2341, 2020)

(DOI: 10.2169/internalmedicine.4721-20)

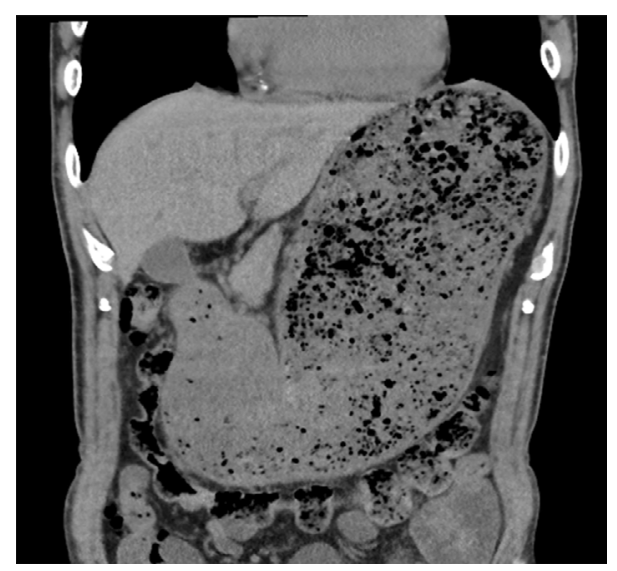

Picture 1.

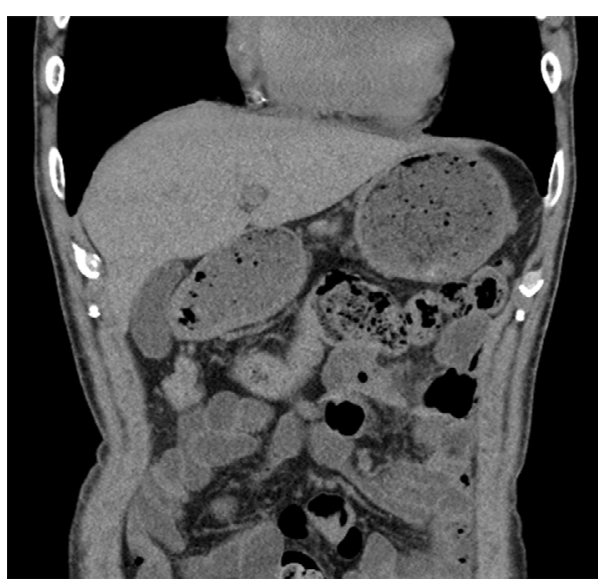

Picture 2.
A 71-year-old man with heart failure was admitted for radiofrequency catheter ablation for atrial fibrillation. Pulmonary vein isolation (PVI) and cavotricuspid isthmus ablation were successfully performed. Three days after PVI, the patient experienced nausea, and abdominal computed tomography (CT) showed gastric dilation (Picture 1), which improved by day 11 after administration of metoclopramide, mosapride and erythromycin (Picture 2). Catheter ablation can cause gastrointestinal disorders through periesophageal vagal nerve injury due to the proximity to the pulmonary vein; its incidence within 48 hours of the procedure is $1.1 \%$ (1). Gastric dilation is often diagnosed by CT, and gastric peristalsis is confirmed by endoscopy. Peristaltic stimulants are useful for improving gastric motility (1). Esophageal temperature monitoring during catheter ablation can prevent vagal nerve injury (2). Although most abdominal symptoms improve noninvasively, some cases require surgery (2). Gastric peristalsis due to esophageal plexus injury should be considered as a cause of abdominal bloating or nausea after catheter ablation.

The authors state that they have no Conflict of Interest (COI).

\section{References}

1. Shah D, Dumonceau JM, Burri H, et al. Acute pyloric spasm and gastric hypomotility: an extracardiac adverse effect of percutaneous radiofrequency ablation for atrial fibrillation. J Am Coll Cardiol 46: 327-330, 2005.

2. Kuwahara T, Takahashi A, Kobori A, et al. Safe and effective ablation of atrial fibrillation: importance of esophageal temperature monitoring to avoid periesophageal nerve injury as a complication of pulmonary vein isolation. J Cardiovasc Electrophysiol 20: 1-6, 2009.

The Internal Medicine is an Open Access journal distributed under the Creative Commons Attribution-NonCommercial-NoDerivatives 4.0 International License. To view the details of this license, please visit (https://creativecommons.org/licenses/ by-nc-nd/4.0/).

Department of Cardiovascular Medicine, Faculty of Medicine and Graduate School of Medicine, Hokkaido University, Japan Received: February 24, 2020; Accepted: April 21, 2020; Advance Publication by J-STAGE: June 9, 2020 Correspondence to Dr. Takao Konishi, takaokonishi0915@gmail.com

(C) 2020 The Japanese Society of Internal Medicine. Intern Med 59: 2341, 2020 